\title{
Kunst und Curriculum: Zur Bedeutung ästhetischer Lehr-Lern-Prozesse im schulischen Bildungssektor
}

\author{
Micha Fleiner
}

Mike Fleming (2012): The Arts in Education. An Introduction to Aesthetics, Theory and Pedagogy. London: Routledge. ISBN: 978-0-415-62029-1

Gegenwärtige Diskussionen um den Stellenwert der Künste in schulischen Curricula tragen bisweilen polarisierenden Charakter: So verweisen outputorientierte Bildungspragmatiker im Zusammenhang mit künstlerischen Lerninhalten stets auf die grundsätzliche Problematik einer präzisen Leistungsermittlung. Entschiedene Befürworter ästhetischer Lehr-Lern-Prozesse wiederum entgegnen, schulisches Lernen erschöpfe sich nicht in der alleinigen Vermittlung intersubjektiv überprüfbarer Wissensstrukturen, sondern habe vornehmlich der Entwicklung einer schöpferisch-imaginativen Lernerpersönlichkeit zu dienen. Dass - vermeintliche - Dichotomien solcherart heutzutage indes mitnichten als unüberbrückbar zu gelten haben, beweist Mike Flemings jüngst erschienene Monographie (134 S.), in welcher der Verfasser sinnhafte Verbindungslinien zwischen Kunst, Bildung und Lehrplan leserfreundlich aufzuzeigen unternimmt. Das Werk gliedert sich in zehn Kapitel gleichen Umfangs und folgt einer von Beginn an konsequent entwickelten Argumentationslinie.

Bereits in der Einführung (1-7) konfrontiert Fleming den Leser mit der vordringlichen Frage, was unter dem Begriff $>$ Kunst $<$ im Bereich des Unterrichts und der Erziehung letztlich zu verstehen sei. Es gelte, kritisch zu prüfen, ob eine ausschließliche Orientierung an etablierten Kunstgattungen im engeren Sinne wie Musik, Bildende Kunst, Theater und Tanz die symbolisch zunehmend verdichteten Gefühls- und Erfahrungswelten junger Schülerinnen und Schüler angemessen zu repräsentieren vermag, oder ob zeitgemäße Schulcurricula Lernenden darüber hinaus nicht die Möglichkeit eröffnen sollten, mit künstlerischen Ausdrucksfeldern wie Architektur, Skulptur, Video- und Filmkunst, Graffiti oder Body Art in Berührung zu kommen. ${ }^{1}$ Ohne der Versuchung zu erliegen, hierauf eine vorschnelle Antwort zu erteilen, richtet der Verfasser

\footnotetext{
${ }^{1}$ Allein am Beispiel des schulischen Musikunterrichts, der „neben Oper, Lied und Programmmusik [...] heute auch Performance, Klangkunst, Videoclip, Live-Acts in Pop und Rock, Computeranimation oder Sound Design" (Gies 2009: 145) problemlos zu integrieren weiß, wird deutlich, dass ein eng gefasster Kunstbegriff im modernen Bildungsbereich an seine inhaltlichen Grenzen zu stoßen scheint.
} 
sein Augenmerk zunächst auf die Entwicklung einer argumentativen Legitimationsgrundlage für den Einsatz der Künste in pädagogischen Kontexten (8-19). Hieran anknüpfend verortet Fleming den Begriff 'Kunst< im theoretischen Diskurs (20-32): Angesichts seines umfassenden Sinngehaltes wird dieser Schlüsselbegriff zu definitorisch grenznahen Theoriekonzepten wie >Darstel-

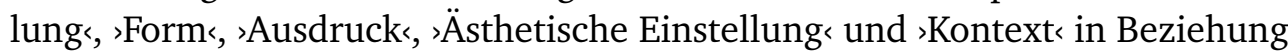
gesetzt. Die Darlegung ist wohlbegründet, allein im Zuge einer begrifflichen Abgrenzung gegenüber der `Ästhetik überrascht, dass maßgebliche Autoren des postmodernen Ästhetik-Diskurses - man denke an Lyotard, Derrida oder Foucault - gänzlich unerwähnt bleiben. ${ }^{2}$

Im dritten Kapitel (33-46) analysiert der Verfasser unterschiedliche Faktoren, die - retrospektiv betrachtet - maßgeblichen Einfluss auf die Vermittlung der Künste im Bildungswesen ausüben sollten. Einen weiten historischen Bogen von Rousseau über Pestalozzi bis Dewey spannend, wird die Entwicklung zentraler pädagogischer Impulse beschrieben, deren Grundgedanken sich in vielfältiger Weise auch in aktuellen Konzeptionen ästhetischer Bildung widerspiegeln. Im Zusammenhang mit Erläuterungen zu kulturtheoretischen Ansätzen spricht sich Fleming mit Entschiedenheit gegen eine didaktische Hierarchisierung in erst- und zweitklassige Kunstformen aus, da der Prozess der ästhetischen Urteilsfindung in hohem Grade an die Gefühlswelt des selektiv wahrnehmenden Subjektes gebunden sei. Vor dem Hintergrund einer stärkeren Subjektorientierung in künstlerischen Lehr-Lern-Prozessen erscheint es konsequent, dass der Autor im Folgenden (47-57) die grundsätzliche Frage nach einer veränderten Verbindung zwischen Kunst und Wissenschaft aufgreift. Auf der Basis des aktuellen Forschungsdiskurses legt er dar, auf welchem Wege ein gemeinhin nach Synthese strebendes Selbstverständnis der Künste mit einem primär analytisch ausgerichteten Forschungsinteresse traditioneller Wissenschaften in Einklang gebracht werden könne:

Being able to see things from different perspectives and being encouraged to notice things is precisely what our involvement in the arts can develop. It is no surprise therefore that the arts are increasingly being used in the process of research itself. (51)

Im Anschluss hieran wird der Akzent auf die historisch mitunter wechselvolle Entwicklung der Künste gelegt (58-67). Anhand eines Rückgriffs auf bedeutende geistesgeschichtliche Epochen sucht der Verfasser, Konsequenzen für die Ausbildung eines geschichtlich gewachsenen Selbstverständnisses der Künste abzuleiten. Fleming verdeutlicht dies, indem er modellhaft deren Implementierungsprozess im Schul- und Erziehungswesen Großbritanniens nachzeichnet.

Durch die Gegenüberstellung zweier Lernkonzepte - dem intrinsisch ausgerichteten „learning in the arts" (68, Hervorhebung im Original) sowie dem extrinsisch motivierten „learning through the arts" (ebd., Hervorhebung im

\footnotetext{
${ }^{2}$ Vgl. hierzu ausführlich Welsch (2010: 42-45); zum dynamischen Wechselverhältnis von ,Ästhetik` und `Kunstく in der Postmoderne vgl. ferner Welsch (2012).
} 
Original) - verfolgt der Autor die Absicht, eine Brücke zwischen Theorie und Praxis zu schlagen. Es ist begrüßenswert, dass er sich nicht mit einer deskriptiven Gegenüberstellung begnügt, sondern - den Blick fortwährend auf praxisrelevante Konsequenzen für den Einsatz der Künste im Bildungssektor gerichtet - eine konzeptuelle Synthese anzustreben sucht:

[...] these perceptions can be balanced by seeing the positive contribution from each side. The 'learning through' approach has potential to challenge teachers to think about purpose and aims without these becoming narrow and instrumental. In this approach, art is removed from its pedestal and embedded in real-life contexts. The 'learning in' view when considered in a more balanced way contains an implicit argument for maintaining the concept of arts in education and resisting the implicit tendency to dissolve the concept of art into creativity and culture. (77)

Es folgen theoriebasierte Ausführungen zum Begriff der `Kreativität< (78-85): Schrittweise führt Fleming den Leser in die Definitionsproblematik ein und legt plausibel dar, welche Chancen bzw. Risiken eine Übertragung des Konzeptes ,Kreativität auf den Bereich der schulischen Bildung birgt.

Einer Fragestellung von gesteigerter Relevanz, da für den wissenschaftlichen Diskurs ein beträchtliches Maß an Konfliktstoff enthaltend, stellt sich der Autor im achten Kapitel seines Werkes (86-95): In welchem Umfang scheinen Verfahren der Leistungsmessung bzw. -bewertung geeignet, die Qualität künstlerischer Arbeitsprozesse adäquat wiederzugeben? Fleming skizziert Lösungsansätze, doch bewegen sich seine Darstellungen hierzu in einem recht allgemeinen Rahmen. An dieser Stelle hätte man sich einen verstärkten Fokus auf konkrete Evaluationsmöglichkeiten für die unterrichtliche Praxis gewünscht, die insbesondere den spezifischen Bewertungskontext der jeweiligen künstlerischen Einzeldisziplin in gebührender Weise berücksichtigen.

Bevor dem Leser wesentliche Aspekte aus den vorangehenden Kapiteln in einem argumentativen Gesamtzusammenhang (110-118) dargeboten werden, widmet sich der Verfasser einer Darstellung (96-109) ausgewählter künstlerischer Teilbereiche (Bildende Kunst, Erzählliteratur, Schauspiel, Musik, Tanz, Lyrik). Dass es sich bei der getroffenen Auswahl um eine Zusammenstellung offenen Charakters handelt, die eine Ergänzung weiterer Einzeldisziplinen prinzipiell nicht ausschließt, ist selbstverständlich. Gerne hätte man an dieser Stelle noch mehr über die einzelnen Unterrichtsfächer des ästhetischen Bereichs und ihre lehrplanübergreifenden Vernetzungsmöglichkeiten erfahren. In Anbetracht des knapp bemessenen Artikelumfangs muss sich der Leser hierbei jedoch mit einer überblickhaften Beschreibung bescheiden.

Ungeachtet der Tatsache, dass sich der überwiegende Teil des Buches auf die bildungspolitischen Rahmenbedingungen Großbritanniens bezieht, lassen sich entscheidende Gedankengänge Flemings ohne Weiteres auf das Bildungssystem im deutschsprachigen Raum übertragen. Denn - vor diesem Hintergrund darf dies Erwähnung finden - auch hierzulande ist eine Aufwertung der Künste im Bereich des (hoch)schulischen Bildungssektors insofern dringend erforderlich, als künstlerische Fachinhalte trotz einer kompetenzorientierten Wende und 
damit verbundener curricularer Reformen bedauerlicherweise nach wie vor einem offenkundigen Marginalisierungsprozess zu unterliegen scheinen.

Fazit: Mike Fleming liefert mit der vorliegenden Monographie einen kritisch abwägenden, durchweg verständlichen Einblick in das facettenreiche Themengebiet der Künste im Bildungswesen. Die wohldurchdachte Gliederung des Werkes findet ihre Entsprechung in einer stringenten Argumentationskette, welche die Leserschaft mühelos durch die zentralen Themenfelder zu geleiten vermag. Besonderes Verdienst gebührt der Einbettung einer Vielzahl an disziplinübergreifenden Praxisbeispielen aus den Bereichen Musik, Literatur, Bildende und Darstellende Kunst, welche theoretisch vielschichtige Sachverhalte in kondensierter Form verdeutlichen. Leserinnen und Leser mit Interesse an einer inhaltlich vertieften Lektüre seien zudem auf die sorgfältig recherchierten Hinweise zu weiterführender Literatur am Ende eines jeden Kapitels verwiesen. Neben Lehramtsstudierenden, Lehrkräften, Erziehungs- und Bildungswissenschaftler/innen mit Interesse am ästhetischen Fächerverbund, sei das vorliegende Werk auch allen bildungspolitischen Entscheidungsträgern empfohlen, die sich - im Zeichen eines ganzheitlich ausgerichteten Bildungswesens - für die Verbreitung künstlerischer Ideen zu engagieren bereit sind.

\section{Bibliografie}

Fleming, Mike (2012): The Arts in Education. An Introduction to Aesthetics, Theory and Pedagogy. London: Routledge

Gies, Stefan (2009): Bilder öffnen Ohren. Musik in Verbindung mit Bild und Szene. In: Jank, Werner (Hg.): Musik-Didaktik. Praxishandbuch für die Sekundarstufe I und II. 3. Aufl. Berlin: Cornelsen Scriptor, 145-151

Welsch, Wolfgang (2010): Ästhetisches Denken. Stuttgart: Reclam Welsch, Wolfgang (2012): Blickwechsel. Neue Wege der Ästhetik. 7. Aufl. Stuttgart: Reclam 